\title{
THE GRONWALL INEQUALITY FOR MODIFIED STIELTJES INTEGRALS ${ }^{1}$
}

WAYNE W. SCHMAEDEKE AND GEORGE R. SELL

1. Introduction. It is well known [1] that if $u$ and $v$ are nonnegative integrable functions and $\epsilon>0$ and if

$$
u(t) \leqq \epsilon+\int_{0}^{t} u(s) v(s) d s, \quad(0 \leqq t \leqq T),
$$

then

$$
u(t) \leqq K \epsilon, \quad(0 \leqq t \leqq T),
$$

where $K=\exp \int_{0}^{T} v(s) d s$. It is the purpose of this paper to investigate the analogous result for modified Stieltjes integrals. We shall show that under appropriate conditions the Gronwall inequality (2) does hold for the modified Stieltjes integrals we consider; however, it does not hold in general. In fact the integral equation

$$
f(t)=\int_{0}^{t} f(s) d g(s)
$$

may have nontrivial positive solutions (cf. [6]).

The inequality we shall obtain is of importance in studying the existence of solutions to certain problems in optimal control theory [5]. Moreover, it is connected with an eigenvalue problem for mean Stieltjes integrals which we discuss briefly in $\$ 3$.

2. Definition of the modified Stieltjes integrals. A subdivision $\sigma$ of an interval $[a, b]$ is defined to be a finite set of numbers $x_{0}, x_{1}$, $\cdots, x_{n}$ with $a=x_{0}<x_{1}<\cdots<x_{n}=b$. Any subdivision $\sigma_{1}$ is said to be a refinement of $\sigma$ if $\sigma \subset \sigma_{1}$. Let $f$ and $g$ be defined on $[a, b]$ and let $\sigma$ be a subdivision of $[a, b]$. Let $S_{\sigma}(f, g)$ denote the sum

$$
S_{\sigma}(f, g)=\sum_{i} \frac{1}{2}\left[f\left(x_{i}\right)+f\left(x_{i-1}\right)\right]\left[g\left(x_{i}\right)-g\left(x_{i-1}\right)\right] .
$$

The integral $\int_{a}^{b} f(s) d g(s)$ is defined to be "limit" of $S_{\sigma}(f, g)$. More precisely, if there is a real number $I$ with the property that for every $\epsilon>0$ there is a subdivision $\sigma$ of $[a, b]$ such that for any refinement $\sigma_{1}$ of $\sigma$ one has $\left|S_{\sigma_{1}}(f, g)-I\right|<\epsilon$, then we define $I$ to be the integral

Received by the editors April 20, 1967.

1 Supported in part by NSF Grant GP-3904. 
$\int_{a}^{b} f(s) d g(s)$. This integral is known as the mean $\sigma$-integral. Another integral, the Dushnik-integral, will also be of interest to us. It differs from the mean $\sigma$-integral in that the term $\frac{1}{2}\left[f\left(x_{i}\right)+f\left(x_{i-1}\right)\right]$ is replaced by $f\left(\xi_{i}\right)$, where $\xi_{i}$ is any point that satisfies $x_{i-1}<\xi_{i}<x_{i}$. We refer the reader to [2], [3], and [4] for further details on these two integrals. We shall use the symbol $(m) \int_{a}^{b} f(s) d g(s)$ and $(b) \int_{a}^{b} f(s) d g(s)$ to refer to the mean $\sigma$ - and the Dushnik-integrals, respectively.

It is known that if $f$ is a function with only discontinuities of the first kind and $g$ is a function of bounded variation, then both the mean $\sigma$ - and the Dushnik-integrals exist. The importance of these two integrals is a consequence of the following representation theorem, which is an extension of the Riesz Theorem. Let $S$ denote the space of all real-valued functions defined on $[a, b]$ with right- and left-hand limits at every point in $[a, b]$. Define a norm on $S$ by $\|f\|_{\infty}=\sup \{|f(s)|: a \leqq s \leqq b\} . S$ is then a Banach space. The following theorem gives a representation for bounded linear functionals on $S$.

THEOREM. Let l be a continuous linear functional on the space $S$. Then there exist functions of bounded variation $g$ and $h$ such that for every $f$ in $S$ one has

$$
l(f)=(m) \int_{a}^{b} f(s) d g(s)+(b) \int_{a}^{b} f(s) d h(s) .
$$

The proof of this theorem is a direct consequence of a theorem of Kaltenborn (cf. [4]) and we shall omit the details.

In the sequel we shall be primarily concerned with the mean $\sigma$ integral. Before discussing the Gronwall inequality let us make note of the following properties:

For any functions $f$ and $g$ of bounded variation where $g$ is right continuous, one has

$$
\begin{aligned}
& \lim _{b \rightarrow a^{+}}(m) \int_{a}^{b} f(s) d g(s)=0, \\
& \lim _{a \rightarrow b^{-}}(m) \int_{a}^{b} f(s) d g(s)=\frac{1}{2}\left[f(b)+f\left(b^{-}\right)\right] \triangle g(b),
\end{aligned}
$$

where $\Delta g(b)=g(b)-g\left(b^{-}\right)$. The first limit shows that the integral

$$
(m) \int_{a}^{t} f(s) d g(s)
$$

is right continuous in $t$. Another property we need is 


$$
(m) \int_{a}^{b} f(s) d g(s) \leqq \sup \{|f(s)|: a \leqq s \leqq b\} V[g ; a, b]
$$

where $V[g ; a, b]$ is the total variation of $g$ on the interval $[a, b]$.

3. The Gronwall inequality. We can now state our main result.

MaIN TheOREM. Let $f$ and $g$ be functions of bounded variation on $[0, T]$ and let $\epsilon \geqq 0$. Assume further that $f$ and $g$ are right continuous and that $f \geqq 0$ and $g$ is increasing. If

$$
f(t) \leqq \epsilon+(m) \int_{0}^{t} f(s) d g(s), \quad 0 \leqq t \leqq T,
$$

then there exist constants $T^{\prime}$ and $K$, depending on $g$ but not $f$, such that $0<T^{\prime} \leqq T, 0 \leqq K$ and

$$
f(t) \leqq K \epsilon, \quad 0 \leqq t<T^{\prime},
$$

Furthermore, $T^{\prime}$ is maximal in the sense that either $T^{\prime}=T$ or $\Delta g\left(T^{\prime}\right) \geqq 2$.

Corollary 1. Assume that the hypotheses of the above theorem are satisfied and $V[g ; 0, T]<2$. Then $T^{\prime}=T$ and (5) holds on $0 \leqq t \leqq T$.

Corollary 2. Assume that the hypotheses of the above theorem are satisfied and that $f$ is continuous. Then $T^{\prime}=T$ and (5) holds on $0 \leqq t \leqq T$.

Before proving the theorem, let us show that $T^{\prime}$ is maximal in the sense indicated. Let $g$ be an increasing function with $\Delta g(\tau)=b \geqq 2$, where $0<\tau \leqq T$ and let

$$
\begin{aligned}
f(t) & =0, & & 0 \leqq t<\tau, \\
& =1, & & \tau \leqq t \leqq T .
\end{aligned}
$$

Since $g$ is increasing one has $g(t) \geqq g(\tau)$ for $t \geqq \tau$. By direct computation one has

$$
\text { (m) } \begin{aligned}
\int_{0}^{t} f(s) d g(s) & =0, \quad 0 \leqq t<\tau, \\
& =g(t)-g(\tau)+b / 2, \quad \tau \leqq t \leqq T .
\end{aligned}
$$

Hence $f(t) \leqq(m) \int_{0}^{t} f(s) d g(s)$, that is (4) is satisfied with $\epsilon=0$, however (5) holds only for $0 \leqq t<\tau$.

We now proceed to the proof. Since $f$ and $g$ are functions of bounded variation there exist constants $B$ and $M$ such that $|f(t)| \leqq B$ on $[0, T]$ and $V[g ; 0, T]=M$. We shall assume that the hypotheses of the above theorem hold throughout. 
Leмma 1. If $f(t) \leqq K \epsilon$ for $0 \leqq t \leqq t_{1}$, then there is a $t_{2}>t_{1}$ and a $K^{\prime}$ such that $f(t) \leqq K^{\prime} \epsilon$ for $0 \leqq t \leqq t_{2}$.

Proof. Since $g$ is right continuous we can find a $t_{2}>t_{1}$ such that $V\left[g ; t_{1}, t_{2}\right]=\rho<1$. Then for $t_{1} \leqq t \leqq t_{2},(4)$ becomes

$$
\begin{aligned}
f(t) & \leqq \epsilon+(m) \int_{0}^{t_{1}} f(s) d g(s)+(m) \int_{t_{1}}^{t_{2}} f(s) d g(s) \\
& \leqq \epsilon+K \epsilon M+B \rho .
\end{aligned}
$$

By replacing the bounded $B$ with $(K M+1) \epsilon+B \rho$ in (6) we get

$$
f(t) \leqq(K M+1) \epsilon(1+\rho)+B \rho^{2} .
$$

By reiterating this we conclude that

$$
f(t) \leqq(K M+1)(1-\rho)^{-1} \epsilon, \quad\left(t_{1} \leqq t \leqq t_{2}\right),
$$

which completes the proof of Lemma 1.

LEMma 2. If $f(t) \leqq K \epsilon$ for $0 \leqq t \leqq t_{1}$ and $V\left[g ; t_{1}, t\right] \leqq \rho<1$ for $t_{1} \leqq t<t_{2}$, then there is a $K^{\prime}$ such that $f(t) \leqq K^{\prime} \epsilon$ for $0 \leqq t<t_{2}$.

This follows from the observation that the estimates in Lemma 1 are uniform for $t_{1} \leqq t<t_{2}$. Since $g$ is right continuous one should note that the hypothesis on the variation in Lemma 2 can be satisfied even if $V\left[g ; t_{1}, t_{2}\right] \geqq 1$.

Leмma 3. If $f(t) \leqq K \epsilon$ for $0 \leqq t<t_{1}$ and $\triangle g\left(t_{1}\right)<2$, then there is a $K^{\prime}$ such that $f(t) \leqq K^{\prime} \epsilon$ for $0 \leqq t \leqq t_{1}$.

Proof. We want to compute a bound for $f\left(t_{1}\right)$. If $0 \leqq \tau<t_{1}$, then one has

$$
\begin{aligned}
f\left(t_{1}\right) & \leqq \epsilon+(m) \int_{0}^{t_{1}} f(s) d g(s) \\
& =\epsilon+(m) \int_{0}^{\tau} f(s) d g(s)+(m) \int_{\tau}^{t_{1}} f(s) d g(s) \\
& \leqq \epsilon+K \epsilon V[g ; 0, \tau]+(m) \int_{\tau}^{t_{1}} f(s) d g(s) .
\end{aligned}
$$

Now let $\tau \rightarrow t_{\overline{1}}$. From (3) we get

$$
f\left(t_{1}\right) \leqq \epsilon+K M \epsilon+\frac{1}{2}\left[f\left(t_{1}\right)+f\left(t_{1}^{-}\right)\right] \triangle g\left(t_{1}\right) .
$$

Since $f\left(t_{\overline{1}}\right) \leqq K \epsilon,(8)$ becomes 


$$
f\left(t_{1}\right) \leqq(K M+K+1) \epsilon+\frac{1}{2} f\left(t_{1}\right) \triangle g\left(t_{1}\right),
$$

or

$$
f\left(t_{1}\right) \leqq \frac{K M+K+1}{1-\frac{1}{2} \triangle g\left(t_{1}\right)} \epsilon .
$$

Proof of Main Theorem. First define $T^{\prime}$ to be either the first point (in the natural ordering) for which $\Delta g\left(T^{\prime}\right) \geqq 2$, or if no such point exists, set $T^{\prime}=T$. In the interval $\left[0, T^{\prime}\right)$ list all the points $T_{1}, T_{2}, \cdots, T_{n}$ for which $1 \leqq \Delta g\left(T_{i}\right)<2, i=1,2, \cdots, n$. (Since $g$ has bounded variation there are at most a finite number of these.)

Now consider the interval $\left[0, T_{1}\right]$. Since $g$ has bounded variation we can find a real number $\rho$ and a partition of the interval $\left[0, T_{1}\right]$, $0=t_{0}<t_{1}<\cdots<t_{m}<T_{1}$ so that $0<\rho<1$ and

$$
\begin{aligned}
V\left[g ; t_{i-1}, t_{i}\right] & \leqq \rho, \quad(i=1,2, \cdots, m) \\
V\left[g ; t_{m}, t\right] & \leqq \rho, \quad\left(t_{m} \leqq t<T_{1}\right) .
\end{aligned}
$$

Now apply Lemma 1 successively to each of the intervals $\left[t_{i-1}, t_{i}\right]$ and Lemma 2 to the interval $\left[t_{m}, T_{1}\right)$. One then has $f(t) \leqq K \epsilon$ on $0 \leqq t<T_{1}$. Now apply Lemma 3 thereby getting $f(t) \leqq K^{\prime} \epsilon$ on $0 \leqq t \leqq T_{1}$. By repeating this process for each of the intervals $\left[T_{1}, T_{2}\right], \cdots$, $\left[T_{n}, T^{\prime}\right)$ we complete the proof of the theorem. It should be noted that the final constant $K$ is finite and it can be computed directly by applying (7) and (9) a finite number of times.

It should be clear from the above that if $T^{\prime}=T$ and $\Delta g(T)<2$, then the inequality (5) can be satisfied on the closed interval $0 \leqq t \leqq T$.

Proof of Corollaries. The first corollary is obvious since for every $\tau, 0 \leqq t \leqq T$, one has $\triangle g(\tau) \leqq V[g ; 0, T]$. To prove the second corollary we simply refer back to Lemma 3 and note that if $f$ is continuous one does not need the assumption that $\Delta g\left(t_{1}\right)<2$, to get the desired conclusion.

REMARKs. 1 . The pathology that occurs when $\triangle g\left(T^{\prime}\right) \geqq 2$ for the mean $\sigma$-integral does not arise for the Dushnik-integral. One can show that if

$$
f(t) \leqq \epsilon+(b) \int_{0}^{t} f(s) d g(s), \quad(0 \leqq t \leqq T),
$$

where $f, g$ and $\epsilon$ are as given in the Main Theorem, then there is a constant $K$ such that $f(t) \leqq K \epsilon,(0 \leqq t \leqq T)$.

The argument for the Dushnik-integral differs in only one spot from that given above; viz. inequality (8) now becomes 


$$
f\left(t_{1}\right) \leqq \epsilon+K M \epsilon+f\left(t_{1}\right) \triangle g\left(t_{1}\right) .
$$

This now implies that

$$
f\left(t_{1}\right) \leqq\left(K M+K \triangle g\left(t_{1}\right)+1\right) \epsilon,
$$

regardless of the size of $\triangle g\left(t_{1}\right)$.

2. If $f$ satisfies an inequality of the form

$$
|f(t)| \leqq \epsilon+(m) \int_{0}^{t}|f(s)| k(s) d g(s), \quad 0 \leqq t \leqq T .
$$

This can be reduced to case studied in the Main Theorem by replacing (10) with

$$
|f(t)| \leqq \epsilon+(m) \int_{0}^{t}|f(s)| d v(s),
$$

where $v(t)=(m) \int_{0}^{t} k(s) d g(s)$.

3. The failure of Gronwall's inequality when $\triangle g\left(T^{\prime}\right) \geqq 2$ is related to the eigenvalue problem

$$
\lambda f(t)=(m) \int_{0}^{t} f(s) d g(s) .
$$

That is, if we defined the operator

$$
U: f \rightarrow(m) \int_{0}^{t} f(s) d g(s), \quad 0 \leqq t \leqq T,
$$

one can show that $\lambda(\lambda>0)$ is an eigenvalue of $U$ if and only if there is a $T^{\prime}, 0<T^{\prime} \leqq T$ such that $\Delta u\left(T^{\prime}\right)=2 \lambda$. This subject will be pursued further in a later paper (cf. [6]).

\section{BIBLIOGRAPHY}

1. L. Cesari, Asymptotic behavior and stability problems in ordinary differential equations, 2nd ed., Academic Press, New York, 1963.

2. R. E. Lane, The integral of a function with respect to a function, Proc. Amer. Math. Soc. 5(1954), 59-66.

3. T. H. Hildebrandt, Definitions of Stieltjes integrals of the Riemann type, Amer. Math. Monthly 45 (1938), 265-278.

4. H. S. Kaltenborn, Linear functional operations on functions having discontinuities of the first kind, Bull. Amer. Math. Soc. 40 (1934), 702-708.

5. W. W. Schmaedeke, Optimal control of nonlinear differential equations in which the control enters linearly, J. Optimization Theory (to appear).

6. W. W. Schmaedeke and G. R. Sell, On the existence, uniqueness and continuity of solutions of nonlinear Stieltjes integral equations, (to appear).

UNIVERSITY OF MiNNESOTA 